\title{
Equipos de trauma: realidad mundial e implementación en un país en desarrollo. Descripción narrativa
}

\section{Trauma teams: global reality and implementation in a developing country. Narrative description}

\author{
Francisco Luis Uribe $\mathbb{D}^{\mathbb{D}}$, Sandra Milena Carvajal' ${ }^{2}$, Nicolás Felipe Torres ${ }^{3}$, \\ Luis Alfonso Bustamante ${ }^{4} \mathbb{D}$, Alberto Federico García ${ }^{5} \mathbb{D}$
}

\begin{abstract}
Médico, Asistente de Investigación, Urgencias, Centro de Investigaciones Clínicas, Fundación Valle del Lili, Cali, Colombia. Médico, especialista en Emergencias, Fundación Valle del Lili; profesor titular, Universidad CES, Cali, Colombia.

Médico, residente de Cirugía general, Universidad de Caldas, Manizales, Colombia.

Médico, especialista en Medicina de Emergencias y en Cuidados intensivos, Fundación Valle del Lili; profesor titular, Universidad CES, Cali, Colombia.

5 Médico, especialista en Cirugía general, Cirugía de Trauma y Emergencias, y Cuidado Intensivo; Magister en Epidemiología; profesor titular, Universidad del Valle; Hospital Universitario del Valle; Fundación Valle del Lili, Cali, Colombia.
\end{abstract}

\section{Resumen}

Introducción. El trauma es una de las entidades con mayor morbimortalidad en el mundo. Los equipos especializados en la atención del paciente traumatizado son llamados «equipos de trauma». Dichos equipos surgieron de la necesidad de brindar tratamiento oportuno multidisciplinario a individuos con heridas que condicionan gran severidad en la guerra; sin embargo, con el paso del tiempo se trasladaron al ámbito civil, generando un impacto positivo en términos de tiempos de atención, mortalidad y morbilidad.

El objetivo de este estudio fue describir el proceso de desarrollo de los equipos de trauma a nivel mundial y la experiencia en nuestra institución en el suroccidente colombiano.

Métodos. Se realizó una búsqueda en la base de datos PUBMED, que incluyó revisiones sistemáticas, metaanálisis, revisiones de Cochrane, ensayos clínicos y series de casos.

Resultados. Se incluyeron 41 estudios para esta revisión narrativa, y se observó que el tiempo de permanencia en Emergencias, el tiempo de traslado a cirugía, la mortalidad y las complicaciones asociadas al trauma fueron menores cuando se implementan equipos de trauma.

Discusión. El diseño de un sistema de atención y valoración horizontal de un paciente con traumatismos severos produce un impacto positivo en términos de tiempos de atención, mortalidad y morbilidad. Se hace necesario establecer los parámetros operativos necesarios en las instituciones de salud de alta y mediana complejidad en nuestro país para implementar dichos equipos de trabajo.

Palabras clave: centros traumatológicos; heridas y traumatismos; índices de gravedad del trauma; atención de apoyo vital avanzado en trauma; equipo hospitalario de respuesta rápida; grupo de atención al paciente.

Fecha de recibido: 07/05/2020 - Fecha de aceptación: 17/06/2020

Correspondencia: Francisco Luis Uribe. Carrera 98 \# 18-49, Centro de Investigaciones Clínicas, Fundación Valle del Lili, Cali, Colombia. Teléfono: (2) 3319090. Correo electrónico: francisco.uribe@fvl.org.co

Citar como: Uribe FL, Carvajal SM, Torres NF, Bustamante LA, García AF. Equipos de trauma: realidad mundial e implementación en un país en desarrollo. Descripción narrativa. Rev Colomb Cir. 2021;36:42-50. https://doi.org/10.30944/20117582.650

Este es un artículo de acceso abierto bajo una Licencia Creative Commons - BY-NC-ND https://creativecommons.org/licenses/by-ncnd/4.0/deed.es 


\begin{abstract}
Introduction. Trauma is one of the entities with the highest morbidity and mortality in the world. Teams specialized in trauma patient care are called «trauma teams». These teams arose from the need to provide timely multidisciplinary treatment to individuals with severe injuries in war; however, with time they moved to the civilian arena, generating a positive impact in terms of care times, mortality and morbidity. The objective of this study was to describe the process of development of trauma teams worldwide and the experience in our institution in southwestern Colombia.
\end{abstract}

Methods. A search of the PUBMED database was carried out, which included systematic reviews, meta-analyses, Cochrane reviews, clinical trials, and case series.

Results. Forty-one studies were included for this narrative review, and it was observed that the length of stay in the ER, the time of transfer to surgery, mortality and complications associated with trauma were lower when trauma teams are implemented.

Discussion. The design of a horizontal care and assessment system for a patient with severe trauma produces a positive impact in terms of care times, mortality and morbidity. It is necessary to establish operational parameters in high and medium complexity health institutions in our country to implement such work teams.

Keywords: trauma centers; wounds and injuries; multiple trauma; trauma severity indices; advanced trauma life support care; hospital rapid response team; patient care team.

\section{Introducción}

Según el reporte "Injuries and Violence" emitido por la Organización Mundial de la salud, el $10 \%$ de las muertes a nivel mundial son debidas al trauma, encontrándose dentro de las primeras 10 causas de muerte y aportando 5,8 millones de muertes al año $0^{1,2}$. Las principales causas de muerte secundarias a lesiones de causa externa son accidentes de tránsito ( $24 \%)$, suicidio (16\%), caídas (14\%) y homicidios $(10 \%)^{2}$. Según el Análisis de la Situación en Salud en Colombia (ASIS) en 2016, las lesiones de causa externa fueron la cuarta causa de muerte en la población colombiana, ocasionando el 16,4\% de las muertes, con una alta carga de morbilidad a corto y largo plazo ${ }^{3}$.

El manejo del paciente politraumatizado es un gran reto, y dado su impacto sobre la morbilidad y mortalidad, durante la Primera Guerra Mundial se crearon los primeros equipos de atención en trauma ${ }^{4}$. Esos equipos se enfocaban en el manejo del paciente herido en combate, para brindar la atención inmediata requerida para salvaguardar la vida, buscando atender y trasladar los pacientes, lo antes posible, a centros de mayor complejidad para su manejo definitivo ${ }^{4}$.
Posteriormente, los equipos de trauma fueron trasladados al ámbito civil, y hoy en día, hacen parte fundamental de centros de referencia mundial en manejo de pacientes politraumatizados. Múltiples estudios han demostrado el impacto de la conformación de los equipos de trauma, disminuyendo tiempos de atención, morbilidad y mortalidad ${ }^{4-6}$.

El objetivo de este artículo es hacer una revisión narrativa acerca de las experiencias y desarrollo de estos equipos a nivel mundial y describir la experiencia en el suroccidente colombiano.

\section{Generalidades de equipo de trauma}

Los «equipos de trauma» (o en inglés, trauma teams) son grupos multidisciplinarios cuyo objetivo es brindar una evaluación y manejo oportuno de los pacientes politraumatizados, proporcionar una resucitación oportuna y priorizar el manejo de aquellas lesiones que pueden amenazar la vida del paciente. Están compuestos usualmente por especialistas en medicina de emergencias, anestesiólogos, cirujanos, enfermeros, equipo del banco de sangre y de salas de cirugía, imágenes diagnósticas y apoyo logístico ${ }^{7,8}$. 
Desde los años setenta se crearon los primeros lineamientos legales que guiaron la creación de los actuales protocolos de atención primaria en trauma ${ }^{9}$. Pese a esto, solamente el $20 \%$ de las instituciones cuentan con un equipo de trauma a nivel hospitalario ${ }^{8,10}$.

Existe un consenso en que el líder de la reanimación debe ser el especialista en Medicina de Emergencias o el Cirujano que atiende el primer llamado, apoyado por el Anestesiólogo, como especialista en vía aérea, el personal de enfermería y el técnico en imágenes diagnósticas.

El equipo funciona siguiendo objetivos y roles predefinidos para cada miembro, direccionados por el líder. Usualmente, el médico especialista en Medicina de Emergencias o el Cirujano, es quien se encarga de realizar la valoración inicial ${ }^{7}$. Del mismo modo, realizan la ultrasonografía enfocada al paciente de trauma (FAST, del inglés Focused Abdominal Sonography in Trauma), con el fin de determinar lesiones que amenacen la vida del paciente. El equipo también se compone de dos enfermeros, el primero de ellos encargado del monitoreo de signos vitales y la obtención de monitorización invasiva de la tensión arterial, cuando sea necesario, y el segundo encargado de la preparación y suministro de medicamentos. Finalmente, el técnico de imágenes diagnósticas es el encargado de la toma de radiografías portátiles y la coordinación con la unidad de radiología para la lectura de las mismas ${ }^{7}$.

Es importante no sobrecargar el número de personas que responden al llamado del equipo de trauma; hay estudios que han demostrado que un grupo numeroso no mejora la función del equipo, ni impacta de manera positiva en los desenlaces de los pacientes ${ }^{11}$.

Los estudios han mostrado que la implementación de un equipo de trauma mejora los desenlaces en pacientes politraumatizados, en términos de mortalidad y morbilidad ${ }^{12-16}$. Este impacto se aprecia especialmente en aquellos pacientes con traumas severos. Petrie et al., demostraron que los pacientes con índice de severidad de trauma (RTS) mayor a 12 presentan resultados mejores con la activación de un equipo de trauma versus la atención prestada por personal no especialista en trauma ${ }^{17}$. Cornwell et al., registraron el tiempo que tardaba un paciente politraumatizado en ser llevado desde la sala de emergencias al quirófano, la Unidad de Cuidado Intensivo o la sala de Observación después de la conformación de un equipo de trauma, encontrando una disminución de 84 a 52 minutos, de 197 a 118 minutos y de 300 a 140 minutos, respectivamente, todos con $\mathrm{p}<0,01^{18}$.

Así mismo, Gerardo et al., realizaron un estudio comparativo, antes y después de la implementación del código de trauma en un centro de salud universitario de trauma nivel I, donde encontraron una disminución en la mortalidad global de un $6 \%$ a un $4 \%$, y cuando se analizaron los pacientes con un ISS mayor de 25 puntos, se observó un reducción de la mortalidad de un 30,2 \% a un $22 \%{ }^{19}$. Además, se ha demostrado que cuando la activación del equipo de trauma es realizada para la atención de pacientes con altos índices de severidad de trauma (ISS $>15$ ), el tiempo de traslado de la sala de emergencias a cirugía es menor (170 versus 534 minutos, respectivamente), lo que a su vez lleva a una disminución en términos de mortalidad y complicaciones asociadas al trauma ${ }^{20}$.

La presencia de cirujanos experimentados en traumatismos ha logrado reducir el tiempo de estancia hospitalaria en urgencias de los pacientes con trauma, cerrado y penetrante. Además, para el caso específico del trauma penetrante, ha logrado disminuir el tiempo para las intervenciones quirúrgicas $^{21}$.

\section{Criterios de activación del código de trauma}

Diferentes grupos de trauma han organizado sus protocolos de activación del código de trauma con base en sus experiencias o protocolos institucionales $7,12,15,22$, sin embargo, cada criterio de activación debe ser definido, e idealmente validado, antes de su incorporación al protocolo institucional, con el objetivo de optimizar los recursos e identificar los pacientes que requieren intervención, sin generar una sobre ni subclasificación de los pacientes ${ }^{22,23}$.

El personal del centro de trauma debe estar al tanto de las condiciones clínicas del paciente en el sitio del trauma por medio de la informa- 
ción continua, emitida por el personal de atención prehospitalaria, en tiempo real y veraz ${ }^{12,24}$. Esto funciona así en sistemas de salud desarrollados y más avanzados, donde el sistema de comunicación con el personal de salud prehospitalario está bien estructurado, a diferencia de nuestro medio, donde los sistemas de atención prehospitalaria usualmente no se articulan de manera efectiva con la atención hospitalaria.

Franklin et al., ${ }^{25}$ con base en las variables prehospitalarias, realizaron un análisis de 6976 pacientes traumatizados y documentaron que los pacientes que presentaban hipotensión en la escena prehospitalaria y alcanzaron a llegar con signos de vida a los servicios de emergencia de centros nivel I de trauma, tuvieron una mortalidad del 12 $\%$, comparado con una mortalidad del $32 \%$ de los pacientes que hicieron hipotensión durante su estancia en el servicio de emergencias, sin haber presentado hipotensión en el área prehospitalaria. Dado esto concluyen que la hipotensión prehospitalaria continúa siendo un indicador válido para la activación de los equipos de trauma, incluyendo la presencia de hipotensión durante la estancia en el servicio de emergencias.

Dehli et al., publicaron en el año 2011 la evaluación del rendimiento para detectar cirugías o procedimientos de emergencias (necesidad de intubación orotraqueal, tubo a tórax, cirugía hemostática en abdomen, cirugía hemostática en pelvis con empaquetamiento, toracotomía, o estabilización primaria de fracturas con fijación externa), y mostraron una sobre-activación del $71 \%$ y una sub-activación del $32 \%$ cuando se valora como estándar de referencia la escala ISS (Injury Severity Score) mayor o igual a 15 puntos, datos que no son muy diferentes cuando se utiliza como estándar de referencia la necesidad de procedimientos de emergencia, con sobre-activación del $71 \%$ y sub-activación del $21 \%{ }^{26}$.

Lehmann et al., ${ }^{27}$ en el año 2007, hicieron una validación de los criterios de activación de trauma del Madigan Army Medical Center de los Estados Unidos, y encontraron que con la aplicación actual de los criterios de activación de código de trauma hay una sobre-clasificación del $51 \%$ y una sub-clasificación del $1 \%$, y mortalidad de $0 \%$ y
$7 \%$, respectivamente. La presencia de hipotensión, dificultad o esfuerzo respiratorio y trauma penetrante en torso se asociaron de manera independiente con la necesidad de intervención de modo emergente.

Para el año 2009, Kouzminova et al., ${ }^{28}$ analizaron la eficacia del sistema de activación del código de trauma de 2 niveles en un centro nivel I de trauma y encontraron diferencia estadísticamente significativa $(p<0,0001)$ entre la activación de trauma mayor versus menor en paciente intubado en el servicio de emergencias $(9,2 \%$ versus $0,1 \%)$, traslado a salas de cirugías $(12,1 \%$ versus $2,3 \%$ ), ingreso a unidad de cuidados intensivos (17,6 \% versus $2 \%$ ), admisión para valoración y observación neurológica (11,6 \% versus 5,9 \%), alta del servicio de urgencias (18,7 \% versus $66 \%)$, muerte en el servicio de urgencias $(2,6 \%$ versus $0 \%)$ y muerte en hospitalización $(6,3 \%$ versus $0,1 \%$ ), entre otros.

En el 2010, Trudi Davis et al., ${ }^{29}$ realizaron un estudio de evaluación prospectivo y durante 12 meses recolectaron información de 1144 activaciones de código de trauma, de los cuales el $41 \%$ fueron activación completa (todo el equipo de trauma involucrando departamentos de emergencias, cirugía, anestesiología, imágenes diagnósticas y otros) y el $52 \%$ fue activación para interconsultas por el grupo de trauma (involucrando únicamente personal del departamento de emergencias y cirugía). En el centro donde se realizó el estudio, el código de trauma de activación completa involucró variables de signos vitales y de lesiones mayores, dejando como criterio para la activación de interconsulta por trauma las variables de cinemática de trauma y aquellos pacientes sin alteraciones de las variables vitales o de lesiones mayores. Este modelo de funcionamiento demostró una sensibilidad del $83 \%$, especificidad de $68 \%$, sub-clasificación de $3 \%$ y sobre-clasificación de $27 \%$, sin evidencia de muertes en el grupo de pacientes de sub-clasificación. Con base en éstos resultados se demuestra una reducción relativa de casi un $50 \%$ y de reducción absoluta de un $33 \%$, comparado con los criterios del American College of Surgeons, para la activación del código de trauma ${ }^{30}$. 


\section{El trauma en el mundo y Colombia}

Las lesiones traumáticas acarrean con un $11 \%$ de la tasa mundial de mortalidad y representan el $10 \%$ de la carga de enfermedad asociada a lesiones traumáticas de todo el mundo ${ }^{31}$. De igual manera, cuando se analizan la carga por discapacidad y mortalidad relacionada al trauma a nivel mundial, los países de ingresos medios y bajos, como Colombia, aportan el $90 \%$ de dicha estadística. También es relevante que, es hasta 6 veces más alta la probabilidad de muerte de un paciente traumatizado en los países de ingresos medios y bajos comparado con los países de altos ingresos, reflejando la probable pobre calidad en cuanto al manejo y cuidados del paciente traumatizado en esta regiones ${ }^{32}$.

En Colombia, la principal causa de muerte reportada por el DANE (Departamento Administrativo Nacional De Estadística) corresponde a enfermedades isquémicas del corazón, seguidas por enfermedades cerebrovasculares, enfermedades respiratorias crónicas y, en cuarto lugar, se encuentran las lesiones de causa externa. Con base en la información sustraída del DANE del año 2018, hubo un total de 228.156 muertes, de las cuales $27.669(12,1 \%)$ fueron violentas. Los departamentos con mayor número de muertes violentas registradas son Antioquia (16,8\%), Valle del Cauca $(14,4 \%)$ y Bogotá $(9,4 \%)^{33}$.

\section{Equipo de trauma en la institución}

Actualmente en nuestro Departamento de Valle del Cauca se cuenta con pocas instituciones con equipos de trauma, dos de las cuales son instituciones de III nivel de complejidad y centros nivel I de trauma, según la clasificación internacional ${ }^{34}$. Adicionalmente, estas instituciones llevan registros de trauma para poder obtener estadísticas que permita conocer el funcionamiento de sus servicios $^{35-38}$. En el 2016 Ordoñez et al., estudiaron la distribución del trauma en la ciudad, basado en los registros de estas dos instituciones, y se recolectó una población de 17.431 pacientes, de los cuales $67 \%$ eran hombres y el grupo etario más afectado estaba entre 18 y 35 años, con una media de 30 años. Los mecanismos más prevalentes de trauma fueron caídas de altura (37 \%), accidentes de trán- sito (12\%) y lesiones por causa violenta (24\%); de estas últimas, las lesiones por arma de fuego fueron el 35\%, arma cortopunzante el 35,5 \% y quemaduras el $10 \%$. Reportaron, además, una mortalidad general de dicha población de 2,5 \%, la cual variaba de acuerdo a la severidad del mismo, según la escala de severidad del trauma, ISS $<15$ puntos, 0,3 \%; ISS 15-24, $34 \%$; ISS > 25, $64 \%{ }^{37,38}$.

En nuestra institución, se conformó el equipo de trauma desde el $1^{\circ}$ de septiembre de 2015. Este equipo de trabajo está constituido por un Especialista en Medicina de Emergencias, que es el líder de la reanimación y quien tiene como función principal dirigir el abordaje del paciente y realizar el FAST. Paralelo a esto, el Cirujano acude a la sala de reanimación al ser activado el código de trauma y es quien, de acuerdo con los hallazgos hemodinámicos, ultrasonográficos y/o imagenológicos, toma la conducta quirúrgica del paciente.

Se cuenta con la intervención de dos Enfermeros, uno de ellos tiene como función aplicar medicamentos, ayudar en accesos vasculares, y apoyar al especialista en la realización de procedimientos. A su vez, el otro Enfermero tiene como función verificar roles, retirar el personal que no realice intervención y apoyar la movilización en bloque. Además, se dispone de 3 auxiliares de enfermería, uno de ellos encargado del registro del código de trauma, y de un terapeuta respiratorio, para apoyo en la vía aérea.

Del mismo, se cuenta con médicos residentes de Medicina de Emergencias e internos, los cuales están en la sala de reanimación brindando apoyo durante el código de trauma. Cada uno de los integrantes del equipo tiene unas funciones asignadas, las cuales se encuentran descritas en una tabla ubicada en el servicio de reanimación en la unidad de urgencias (figura 1).

Con respecto a los criterios de activación del equipo de trauma, cabe mencionar que surgen de consensos de expertos ${ }^{39}$. En nuestra institución, la activación del código de trauma se realiza de acuerdo con el cumplimiento de al menos uno de los criterios de activación (tabla 1) y requiere llamar a una extensión telefónica destinada con este fin. La activación implica el llamado a cirugía de trauma y emergencias, equipos portátiles de imáge- 
nes diagnósticas, banco de sangre, salas de cirugía, servicios de apoyo logístico y guardas de seguridad, los cuales acuden al servicio de reanimación para la intervención (figura 1). Del mismo modo se tiene una extensión telefónica destinada a la solicitud del paquete transfusional. Una vez se determine el tipo de lesión, se estabilice y se defina destino del paciente, se cierra el código de trauma.

Desde el inicio del <<equipo de trauma >> en nuestra institución, el $1^{\circ}$ septiembre del 2015 , hasta el 25 de septiembre del 2019, se han atendido 1305 pacientes; el $85 \%$ fueron hombres, con una media de edad de 28 años. El $45 \%$ aproximadamente fueron víctimas de trauma penetrante $y$ la mayoría presentaban trauma severo, con una mediana de ISS de 19 (rango 11-26). De estos pacientes el 47 \% requirieron intervención quirúrgica por parte de cirugía de trauma y emergencias y al $66 \%$ se le realizo tomografía computarizada de trauma. La mortalidad fue del $13 \%$.

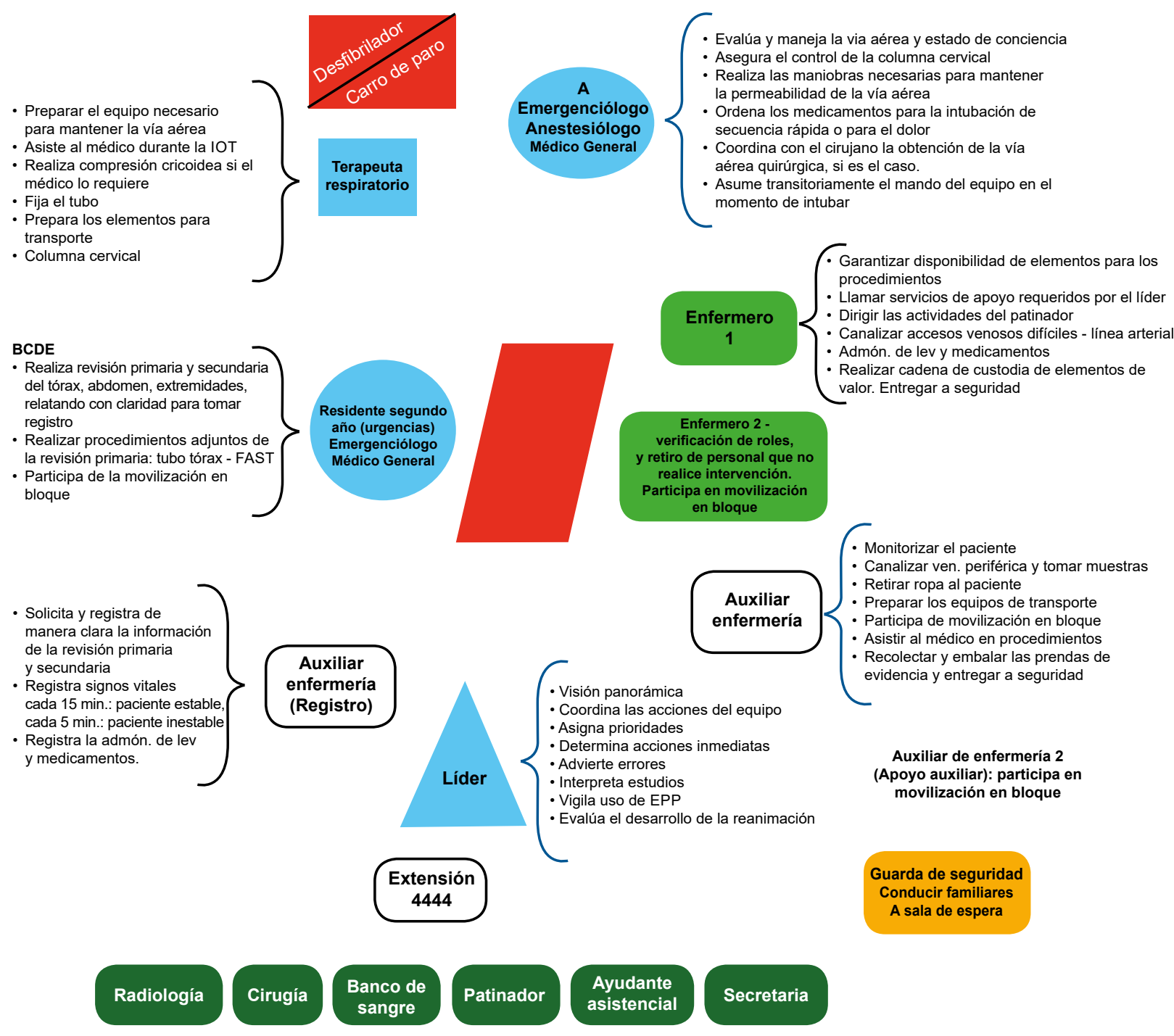

Figura 1. Conformación del Equipo de trauma. Clínica Fundación Valle del Lili, Cali, Colombia. 
Tabla 1. Criterios de activación del Equipo de Trauma. Clínica Fundación Valle del Lili, Cali, Colombia.

\begin{tabular}{l}
\hline \multicolumn{1}{c}{ Criterios activación código de trauma } \\
\hline Paciente intubado \\
Disnea o insuficiencia respiratoria \\
Hipotensión - Presión arterial sistólica (PAS) menor de 90 \\
mmHg \\
Escala de coma de Glasgow menor de 9 \\
Trauma penetrante en torso \\
Amputación traumática \\
Fractura pélvica inestable \\
Paciente previamente operado por cirugía de trauma \\
Víctimas múltiples (2 o más al mismo tiempo) \\
A criterio de quien lo activa
\end{tabular}

\section{Conclusión}

El diseño de un sistema de atención y valoración horizontal de un paciente con traumatismo severo produce un impacto positivo, en términos de tiempos de atención, mortalidad y morbilidad. Por lo tanto, se hace necesario establecer los parámetros operativos requeridos en las instituciones de salud de alta y mediana complejidad en nuestro país, para implementar dichos equipos de trabajo, con el fin de ofrecer las mejores oportunidades a nuestros pacientes en las salas de emergencias, así como propender por el engranaje entre los sistemas prehospitalarios y los centros hospitalarios de baja y mediana complejidad, para poder expandir el radio de acción de estos grupos multidisciplinarios, favoreciendo que más pacientes se beneficien de una atención tan estructurada, como la que brinda los equipos de trauma.

\section{Cumplimiento de normas éticas}

Consentimiento informado. El Comité de Ética Institucional aprobó el diseño y la metodología del estudio. Este estudio es una revisión de la literatura y del registro de trauma retrospectivo, y como tal, no hay necesidad de un consentimiento informado.

Conflictos de interés. Los autores del estudio declaran no presentar conflictos de interés.

Financiación. No se recibió apoyo financiero ni patrocinio externo para este estudio.
Contribución de los autores. Concepción y diseño del estudio, adquisición de datos y redacción inicial del manuscrito: Francisco Luis Uribe, Sandra Milena Carvajal.

Análisis e interpretación de datos, redacción del manuscrito y revisión crítica: Nicolás Felipe Torres, Luis Alfonso Bustamante, Alberto Federico García.

\section{Referencias}

1. World Health Organization. The top 10 causes of death. WHO. 2017. Fecha de consulta: 7 de mayo de 2020. Disponible en:

http://www.who.int/mediacentre/factsheets/fs310/en/

2. World Health Organization. Injuries and violence.The facts 2014. Fecha de consulta: 7 de mayo de 2020. Disponible en: https://apps.who.int/iris/bitstream/ handle/10665/149798/9789241508018_eng.pdf

3. Ministerio de Salud y Proteccion Social. Análisis de Situación de Salud (ASIS) Colombia, 2016. Asis 2016. 2016:1-163. Fecha de consulta: 7 de mayo de 2020. Disponible: https://www.minsalud.gov.co

4. Celso B, Tepas J, Langland-Orban B, Pracht E, Papa L, Lottenberg L, et al. A systematic review and meta-analysis comparing outcome of severely injured patients treated in trauma centers following the establishment of trauma systems. J Trauma Acute Care Surg. 2006;60:371-8. https://doi.org/10.1097/01.ta.0000197916.99629.eb

5. West JG, Trunkey DD, Lim RC. Systems of trauma care. A study of two counties. Arch Surg. 1979;114:455-60. https://doi.org/10.1001/archsurg.1979.013702 80109016

6. West JG, Cales RH, Gazzaniga AB. Impact of regionalization. The Orange County experience. Arch Surg. 1983;118:740-4.

http:/doi.org/10.1001/archsurg.1983.0139 0060058013

7. Tiel Groenestege-Kreb D, Van Maarseveen O, Leenen L. Trauma team. Br J Anaesth. 2014;113:258-265. https://doi.org/10.1093/bja/aeu236

8. Georgiou A, Lockey DJ. The performance and assessment of hospital trauma teams. Scand J Trauma Resusc Emerg Med. 2010;18:66. https://doi.org/10.1186/1757-7241-18-66

9. Shah MN. The formation of the emergency medical services system. Am J Public Health. 2006;96:414-23. https://doi.org/10.2105/AJPH.2004.048793

10. Wong K, Petchell J. Trauma teams in Australia: a national survey. ANZ J Surg. 2003;73:819-25. https://doi.org/10.1046/j.1445-2197.2003.02782.x

11. Driscoll PA, Vincent CA. Variation in trauma resuscitation and its effect on patient outcome. Injury. 1992;23:111-5. https://doi.org/10.1016/0020-1383(92)90044-s 
12. Adedeji OA, Driscoll PA. The trauma team - A system of initial trauma care. Postgrad Med J. 1996;72:587-93. https://doi.org/10.1136/pgmj.72.852.587

13. Hart N, Dasgupta S, Williams EW, Williams-Johnson J. Twenty-four hour trauma team at the University Hospital of the West Indies, Kingston, Jamaica--the time is now. West Indian Med J. 2012;61:211-3. https://doi.org/10.7727/wimj.2012.043

14. Gerardo CJ, Glickman SW, Vaslef SN, Chandra A, Pietrobon $\mathrm{R}$, Cairns CB. The rapid impact on mortality rates of a dedicated care team including trauma and emergency physicians at an Academic Medical Center. J Emerg Med. 2011;40:586-91. https://doi.org/10.1016/j.jemermed.2009.08.056

15. Wuthisuthimethawee P. Trauma team activation criteria in managing trauma patients at an emergency room in Thailand. Eur J Trauma Emerg Surg. 2017;43:53-7. https://doi.org/10.1007/s00068-015-0624-7

16. Cherry RA, King TS, Carney DE, Bryant P, Cooney RN. Trauma team activation and the impact on mortality. J Trauma Inj Infect Crit Care. 2007;63:326-30. https://doi.org/10.1097/TA.0b013e31811eaad1

17. Petrie D, Lane P, Stewart TC. An evaluation of patient outcomes comparing trauma team activated versus trauma team not activated using TRISS analysis. Trauma and Injury Severity Score. J Trauma. 1996;41:870-5. https://doi.org/10.1097/00005373-199611000-00020

18. Cornwell E, Chang C, Phillips J, Campbell K. Enhanced trauma program commitment at a level i trauma center. Effect on the process and outcome of care. Arch Surg. 2003;138:838-43.

https://doi.org/10.1001/archsurg.138.8.838

19. Illescas-Fernández GJ. Escalas e índices de severidad en trauma. Trauma. 2003;6:88-94.

http://www.medigraphic.com/pdfs/trauma/tm-2003/ tm033c.pdf.

20. Wang CH, Hsiao KY, Shih HM, Tsai YH, Chen IC. The role of trauma team activation by emergency physicians on outcomes in severe trauma patients. J Acute Med. 2014;4:1-5. https://doi.org/10.1016/j.jacme.2013.10.006

21. Khetarpal S, Steinbrunn B, McGonigal M, Stafford R, Ney A, Kalb D, et al. Trauma faculty and trauma team activation: impact on trauma system function and patient outcome. J Trauma. 1999;47:576-81.

https://doi.org/10.1097/00005373-199909000-00028

22. Smith J, Caldwell E, Sugrue M. Difference in trauma team activation criteria between hospitals within the same region. Emerg Med Australas. 2005;17:480-7. https://doi.org/10.1111/j.1742-6723.2005.00780.x

23. Rehn M, Lossius HM, Tjosevik KE, Vetrhus M, Østebø O, Eken $\mathrm{T}$ and the Rogaland Trauma System Study Collaborating Group. Efficacy of a two-tiered trauma team activation protocol in a Norwegian trauma centre. $\mathrm{Br}$ J Surg. 2012;99:199-208.

https://doi.org/10.1002/bjs.7794
24. Driscoll PA. Trauma: today's problems, tomorrow's answers. Injury. 1992;23:151-8. https://doi.org/10.1016/S0020-1383(05)80033-6

25. Franklin GA, Boaz PW, Spain DA, Lukan JK, Carrillo EH, Richardson JD. Prehospital hypotension as a valid indicator of trauma team activation. J Trauma. 2000;48:1034-9.

https://doi.org/10.1097/00005373-200006000-00006

26. Dehli T, Fredriksen K, Osbakk SA, Bartnes K. Evaluation of a university hospital trauma team activation protocol. Scand J Trauma Resusc Emerg Med. 2011;19:18. https://doi.org/10.1186/1757-7241-19-18

27. Lehmann RK, Arthurs ZM, Cuadrado DG, Casey LE, Beekley AC, Martin MJ. Trauma team activation: simplified criteria safely reduces overtriage. Am J Surg. 2007;193:630-5. https://doi.org/10.1016/j.amjsurg.2007.01.017

28. Kouzminova N, Shatney C, Palm E, McCullough M, Sherck J. The efficacy of a two-tiered trauma activation system at a level I trauma center. J Trauma. 2009;67:829-33. https://doi.org/10.1097/TA.0b013e3181b57b6d

29. Davis T, Dinh M, Roncal S, Byrne C, Petchell J, Leonard E, Stack A. Prospective evaluation of a two-tiered trauma activation protocol in an Australian major trauma referral hospital. Injury. 2010;41:470-4. https://doi.org/10.1016/j.injury.2010.01.003

30. Centers for Disease Control and Prevention. Guidelines for field triage of injured patients. Recommendations of the National Expert Panel on field triage. Centres Dis Control Prev MMWR. 2012;61:1-21. https://doi.org/10.1542/peds.2009-0257

31. Aboutanos MB, Mora F, Rodas E, Salamea J, Ochoa-Parra M, Salgado E, et al. Ratification of IATSIC/WHO'S guidelines for essential trauma care assessment in the South American region. World J Surg. 2010;34:2735-44. https://doi.org/10.1007/s00268-010-0716-9

32. LaGrone L, Riggle K, Joshipura M, Quansah R, Reynolds T, Sherr K, Mock C. Uptake of the World Health Organization's trauma care guidelines: a systematic review. Bull World Health Organ. 2016;94:585-98C. https://doi.org/10.2471/BLT.15.162214

33. DANE. Defunciones no fetales 2018 preliminar. Fecha de consulta: 12 de mayo de 2020. Disponible en: https://www.dane.gov.co/index.php/estadisticas-por-tema/salud/nacimientos-y-defunciones/defunciones-no-fetales/defunciones-no-fetales-2018

34. Trauma Center Levels Explained - American Trauma Society. Fecha de consulta: 22 de mayo de 2020. Disponible: http://www.amtrauma.org/?page=traumalevels.

35. Ordóñez CA, Pino LF, Tejada JW, Badiel M, Loaiza JH, Mata LV, Aboutanos MB. Experiencia en dos hospitales de tercer nivel de atención del suroccidente de Colombia en la aplicación del Registro Internacional 
de Trauma de la Sociedad Panamericana de Trauma. Rev Col Bras Cir. 2012;39:255-61.

https://doi.org/10.1590/s0100-69912012000400003

36. Gelvez S, Ordoñez CA, Badiel M, Ramirez O, Pino LF, Garcia AF, et al. Evaluación de las escalas ISS y NISS en trauma penetrante grave. Rev Colomb Cir. 2009;24: 229-35.

37. Uribe A, Ordoñez CA, Badiel M, Tejada JW, Loaiza JH, Pino LF, Aboutanos MB. Tendencia del trauma en dos hospitales nivel IV en Cali, Colombia. Reporte preliminar en la plataforma del registro de la Sociedad Pana- mericana de Trauma (SPT/RT). Panam J Trauma, Crit Care Emerg Surg. 2012;1:175-181.

38. Ordoñez CA, Morales M, Rojas-Mirquez JC, Bonilla-Escobar FJ, Badiel M, Arana Miñán F, et al. Registro de Trauma de la Sociedad Panamericana de Trauma: Un año de experiencia en dos hospitales en el suroccidente colombiano. Colomb Med. 2016;47:148-54.

39. Aristizábal D, González G, Suárez JF, Roldán P. Factores asociados al trauma fatal en motociclistas en Medellín, 2005-2008. Biomédica. 2012;32:112-24.

https://doi.org/10.7705/biomedica.v32i1.603 